\title{
Role of physical exercises in common orthopedic conditions
}

\begin{abstract}
Physical exercises are highly effective in common orthopedic conditions like poor posture, chronic neck and back pain due to cervical or lumbar spondylosis, osteoarthritis \& related joint diseases, osteoporosis etc. Exercises are useful in maintaining our muscle and bone mass and its functions. It is associated with happiness and positive mental health
\end{abstract}

Keywords: physical, exercise, osteoporosis, spondylosis, back pain, physiotherapy
Volume 3 Issue 4 - 2018

\author{
Nareshkumar Satyanarayan Dhaniwala \\ Jawaharlal Nehru Medical College, India
}

Correspondence: Nareshkumar Satyanarayan Dhaniwala, Professor of Orthopedics, Jawaharlal Nehru Medical College, DMIMS(DU), Sawangi (Meghe), Wardha 442007, Maharashtra, India, Email nsdhaniwala@gmail.com

Received: June 27, 2018 | Published: September 19, 2018

\section{Introduction}

Physical exercises have an important role in various orthopedic diseases. Exercises could be active, assisted or passive in nature. Each form have its own importance in different conditions. Yogic asanas are also a form of exercise but have to be learnt under guidance of an expert before being undertaken by patients of orthopedic diseases. Physiotherapeutic exercises are relatively easy to learn. Knowledge and practical methods of doing various exercises should definitely be learnt by every orthopedic surgeon for providing complete treatment and training to patients in various orthopedic conditions.

\section{Conditions having utility of exercises}

A large number of orthopedic conditions have proven benefits from physical exercises. Some common conditions are:

1. Traumatic injuries such as ligament sprain, muscle strain and fractures undergoing treatment by surgical or nonsurgical methods. Rehabilitation of a fractured limb or part begins at the time of injury and goes on till the maximum possible functions have been regained. ${ }^{1}$ It consists of joint mobilization, muscle re-education exercises, muscle strengthening exercises and gait training. All modes of exercises are useful in some conditions but the treating surgeon should decide about its optimum use depending on the condition of an individual patient. A discussion with assisting physiotherapist always proves helpful.

2. Back and neck pain due to poor posture are rather common in the present sedentary life style. Regular mobilization exercises of neck, back, upper and lower limb joints along with abdominal muscles help in correction of postural defects and relieves chronic neck or back pain dramatically. It helps in correction of nonstructural alignment defects of spine also. Regarding back muscles there is role of both flexion and extension exercises instead of extension exercises alone.

3. Cervical and lumbar spondylosis involves the middle age population and becomes symptomatic in nearly $40 \%$ to $50 \%$ of people. ${ }^{2}$ These conditions are chronic in nature and can be very disturbing and disabling also. Rest and drugs are useful in its active phase. In chronic situation mobilizing and strengthening exercises against resistance have proved beneficial. Being degenerative in nature spondylosis is associated with stiffness in early morning and after rest. Regular full range mobilization helps in stiffness prevention and makes muscles strong and pliant, fit to stretch in daily activities.

4. Osteoarthritis and other rheumatic diseases have significant benefits due to exercises. Range of movement is retained and improved on regular exercises. Day to today activities become simpler despite arthralgia and deformities. Stiffness is reduced, mobilization of joints and ambulation become easier. Exercises act by pain relief also, as our body releases chemicals called endorphins, which reduce our perception of pain. ${ }^{2}$ Regular knee exercises help a lot in nonsurgical treatment of osteoarthritis despite joint deformities. Mobilization of joints has been shown to help in formation of fibro-cartilage in the areas of damage of articular cartilage. After total joint replacement also exercise of joints is essential.

5. Osteoporosis prevention and treatment: Osteoporosis now is no more a disease of elderly population. It is seen in youth, middle age and elderly alike. It is becoming more common due to indoor life style, less exposure to natural sunlight and lack of exercise, besides dietary deficiency of protein, calcium and vitamins. With aging, bones become very weak and fragile- a condition called osteoporosis. It often occurs in women after menopause, and in men in older age. This bone thinning and weakening disease puts people at a greater risk for broken bones, which can seriously limit mobility and independence. ${ }^{3}$

6. Exercise is important for building strong bones when we are younger, and it is essential for maintaining bone strength when we are older. Exercise works on bones much like it works on muscles-by making them stronger. Bone being a living tissue, it changes in response to forces acting upon it. When we exercise regularly, our bones and muscles adapt by building more cells in bones and more thick and dense fibers in muscles. Weight bearing is a major stimulus for bone formation. ${ }^{4}$ Walking, running and sports provide necessary stimulation for new bone formation. High protein diet, calcium supplementation, anabolic hormones, vitamin $\mathrm{D}$, alendronate are also needed for treatment of osteoporosis.

7. General fitness of body: Exercises of all body parts including 
neck, back, abdomen, chest and limbs done regularly keep our body healthy and joints supple. Exercises release endorphins which gives a feeling of well being and happiness. Exercises for chest causing equal inspiration and expiration maintain the vital capacity of lungs and prevent respiratory infection. Exercises maintain proper metabolism and prevent old agerelated catabolism. Pranayam is considered very useful for lung and heart health. Exercises reduce the risk for heart disease and stroke and prevent obesity. Regular physical activity helps in building and maintaining healthy bones. Regular activity of orthopedic residents on call has been shown to increase fitness level in a study. ${ }^{5}$ Another benefit of exercise is that it improves balance and coordination. This becomes more important in old age because it helps preventing falls and fractures. Exercise has been shown to prevent depression in lonely and elderly persons. Physical exercise has been shown to decrease sarcopenia in elderly people in an Italian study.

\section{Conclusion}

Based on the above benefits of physical exercises it is evident that it is highly important for growth and maintenance of healthy and strong muscles and bones, besides keeping us happy. It prevents osteoporosis and associated fragility fractures. Role of exercise is more in the present era of sedentary life style associated with high prevalence of obesity, osteoporosis, diabetes and hypertension.

\section{Acknowledgments}

None.

\section{Conflicts of interest}

The author declares there are no conflicts of interest.

\section{References}

1. Maheswari J, Mhaskar Vikram A. Treatment of Fractures: General Principles. Essential Orthopedics. 2015. p. 12.

2. Exercise and Depression: Endorphins Release Varies From Exercise Intensity. Dr Mercola's Natural Health Newsletter. 2017.

3. Barbara J Campbell. Exercise and Bone Health. American Academy of Orthopedic Surgeon newsletter. 2012.

4. Peter B Derma, Joseph Liu, Alexander S McLawhorn. 24-hour Fitness: The Orthopedic Resident on-call Workout. HSS J. 2015;11(3):294-298.

5. Giovanni lolascon, Gioconda Di Pietro, Maria Luisa Brandi. Physical exercise and Sarcopenia in older people: position paper of the Italian Society of Orthopedics and Medicine. Clini Cases Miner Bone Metab. 2014;11(3):215-221. 